University of Nebraska - Lincoln

DigitalCommons@University of Nebraska - Lincoln

May 1989

\title{
The influence of crystallographic order upon the electronic structure of thin mercury overlayers
}

\author{
Peter A. Dowben \\ University of Nebraska-Lincoln, pdowben@unl.edu \\ M. Onellion \\ University of Wisconsin, Madison \\ Shikha Varma \\ Syracuse University \\ Y.J. Kime \\ Syracuse University \\ J.L. Erskine \\ University of Texas, Austin, Texas
}

Follow this and additional works at: https://digitalcommons.unl.edu/physicsdowben

Part of the Physics Commons

Dowben, Peter A.; Onellion, M.; Varma, Shikha; Kime, Y.J.; and Erskine, J.L., "The influence of crystallographic order upon the electronic structure of thin mercury overlayers" (1989). Peter Dowben Publications. 135.

https://digitalcommons.unl.edu/physicsdowben/135

This Article is brought to you for free and open access by the Research Papers in Physics and Astronomy at DigitalCommons@University of Nebraska - Lincoln. It has been accepted for inclusion in Peter Dowben Publications by an authorized administrator of DigitalCommons@University of Nebraska - Lincoln. 


\title{
The influence of crystallographic order upon the electronic structure of thin mercury overlayers
}

\author{
P. A. Dowben \\ Department of Physics, Syracuse University, Syracuse, New York 13244-1130 \\ M. Onellion ${ }^{\text {a) }}$ \\ Department of Physics, University of Wisconsin, Madison, Wisconsin 53706 \\ Shikha Varma and Y.J. Kime \\ Department of Physics, Syracuse University, Syracuse, New York, 13244-1130 \\ J. L. Erskine \\ Department of Physics, University of Texas, Austin, Texas 78712
}

(Received 5 August 1988; accepted 10 October 1988)

\begin{abstract}
Thin (1-7 monolayer) well-ordered, disordered, and liquid $\mathrm{Hg}$ overlayers on $\mathrm{Ag}(100)$ have been investigated with angle resolved photoemission and low-energy electron diffraction. The wellordered overlayers exhibit new electronic states as a result of the strained, cubic, overlayer crystallographic structure. The new electronic states depend upon the long-range crystallographic order of the overlayer, not simply the local crystallographic symmetry. The photoemission cross section and branching ratio of the $5 d$ mercury states depends on the overlayer long-range crystallographic order and cannot be explained by photoelectron diffraction.
\end{abstract}

\section{INTRODUCTION}

$\mathrm{Hg}$ overlayers possess three salient advantages for studying the relationship between electronic and crystallographic structure. $\mathrm{Hg}$ is easily adsorbed on $\mathrm{Ag}(100)$ and under certain conditions forms ordered overlayers. The ordered $\mathrm{Hg}$ overlayers, which possess low melting temperatures, permit direct observation of the relationship between the degree of crystallinity and electronic band structure.$^{1-3} \mathrm{Hg}$ overlayers (vide infra) follow the trend reported extensively for the rare-earth elements, ${ }^{4-10}$ where high- $Z$ atoms possess considerable anistropy in the charge distribution and also exhibit an electronic density of states that is sensitive to structure. The complication of the metal-insulator transition, with its attendant change in electronic structure, is not observed for mercury adsorbed on surfaces where it adopts the square lattice of the substrate. ${ }^{11-13}$

\section{EXPERIMENTAL}

Previous reports ${ }^{1-3,13-17}$ of the $\mathrm{Hg}$ on $\mathrm{Ag}(100)$ system have established that $\mathrm{Hg}$ overlayers on $\mathrm{Ag}(100)$ form ordered overlayers. Since the goal of our investigation is the relationship between overlayer long-range crystallographic order and branching ratio, we have studied four overlayer thicknesses: $0.6,1,2$, and 5 monolayers (ML). 1n each case, we measured the integral photoemission intensities from the $\mathrm{Hg} 5 d_{5 / 2}$ and $5 d_{3 / 2}$ levels, which possess binding energies of 8 and $9.8 \mathrm{eV}$ below the Fermi edge, respectively. The branching ratio were calculated from a given energy distribution curve (EDC) by taking the ratio of the $5 d_{5 / 2}$ signal versus the $5 d_{3 / 2}$. This ratio is then plotted as a function of the incident photon energy. For each coverage we observed a new electronic state $e^{1-3,14,16,17}$ for ordered overlayers that is absent for disordered overlayers. A coverage of $0.6 \mathrm{ML}$ is the smallest thickness that exhibits the new electronic state. The new state possesses a binding energy of $\sim 7.2 \mathrm{eV}$ and is included in the $5 d_{5 / 2}$ oscillator strength unless otherwise noted.

The EDC's were obtained using polarized light with the vector potential parallel with the surface. The photoelectrons were collected normal to the surface as described elsewhere. ${ }^{17}$

Long-range order (or lack there of) was ascertained using low-energy electron diffraction. We observe that 1 and $2 \mathrm{ML}$ of $\mathrm{Hg}$ continue the $\mathrm{Ag}(1.00)$ fcc structure (bulk $\mathrm{Hg}$ is rhombohedral), and $5 \mathrm{ML}$ exhibit a cubic $p(1 \times 4)$ ordering. ${ }^{17}$ All overlayers were free of contamination to the limit of our Auger electron spectroscopy system ( 1 at. $\%$ ) and deposited and analyzed as previously described. ${ }^{14,17}$

The experimental apparatus, previously described, ${ }^{14,17}$ was modified by adding a cold stage capable of cooling the $\mathrm{Ag}(100)$ crystal substrate to $30 \mathrm{~K}$. The $\mathrm{Hg}$ overlayers were deposited at substrate temperatures ranging between 30 and $90 \mathrm{~K}$, and subsequently studied over a temperature range of 30 to $200 \mathrm{~K}$. The $\mathrm{Hg}$ overlayer melting temperature was experimentally measured as $150 \mathrm{~K}( \pm 20 \mathrm{~K})$ using a Chromal-Alumel thermocouple. The overlayer thickness was estimated from Auger electron spectroscopy and photoemission data, as described in detail elsewhere. ${ }^{14.17}$

\section{RESULTS AND DISCUSSION}

As shown in the inset to Fig. 1, following the adsorption of 1 and 2 (well-ordered) $\mathrm{ML}$ of $\mathrm{Hg}$ on $\mathrm{Ag}(100)$ at $90 \mathrm{~K}$, three $5 d$-like $\mathrm{Hg}$ features may be identified. ${ }^{14,17}$ For normal emission, these features have binding energies of $9.8,8.0$, and 7.2 $\mathrm{eV}$ with respect to the metal Fermi energy. These features are assigned to the $\mathrm{Hg} 5 d_{3 / 2}(9.8 \mathrm{eV})$, the $\mathrm{Hg} 5 d_{3 / 2}(8.0 \mathrm{eV})$ orbitals, and a third feature believed to be the result of $\mathrm{Hg}$ $\mathrm{Hg}$ orbital interactions (hybridization). ${ }^{1-3,14,17}$ The new electronic states, highlighted with an arrow, are not reported 


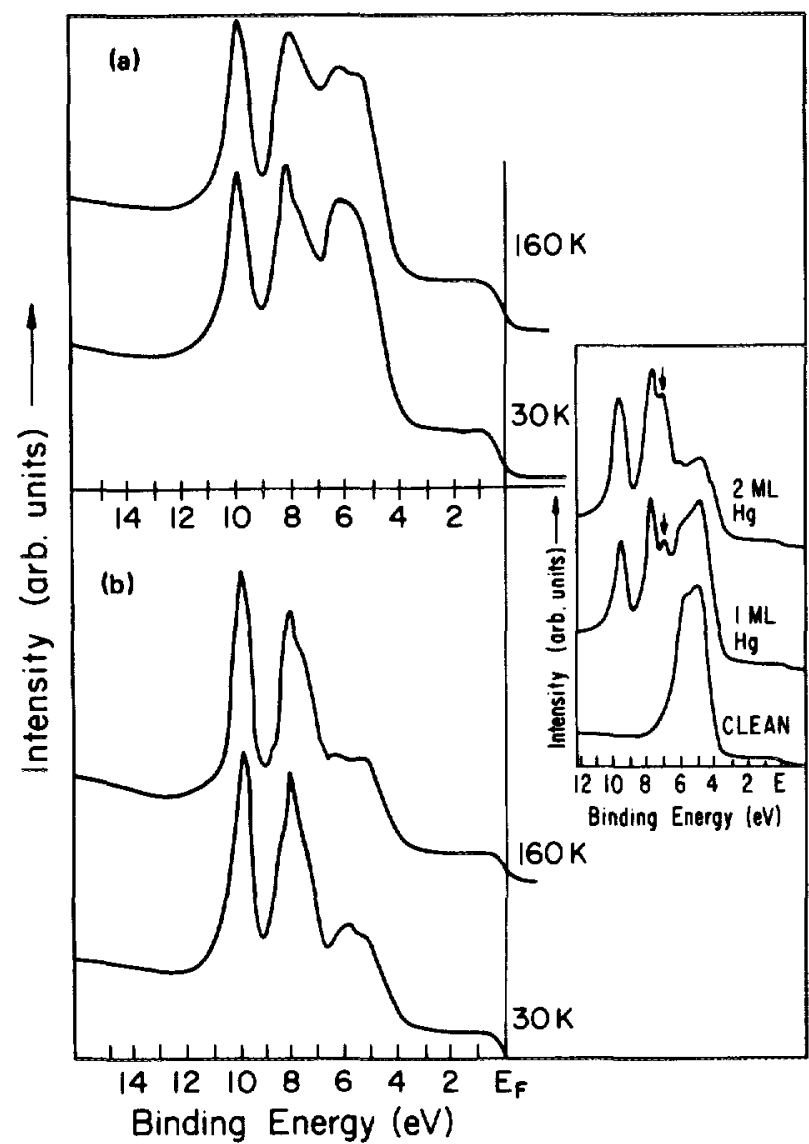

Fig. 1. Angle resolved photoemission spectra for a $\mathrm{Hg}_{\mathrm{g}}$ overlayer deposited on $\mathrm{Ag}(100)$ at $87 \mathrm{~K}$ (insert), at $30 \mathrm{~K}$, and upon annealing the sample to the thin-film melting temperature $(\sim 160 \mathrm{~K})$. The spectra were taken for 1 (a) and 2 (b) $\mathrm{ML}$ of $\mathrm{Hg}$. The photon energy was $50 \mathrm{eV}$, and the electrons were collected normal to the surface with the vector potential of the incident light in the plane of the surface in the $(110\rangle$ direction. The third (new) state believed to be a result of $\mathbf{H g}-\mathbf{H g}$ interactions, observed for the ordered thin films, is indicated with an arrow, in the inset.

for gas phase $\mathrm{Hg}^{18,19}$ One- and two-monolayer $\mathrm{Hg}$ overlayers adsorbed on $\mathrm{Ag}(100)$, at $90 \mathrm{~K}$, adopt the fcc (square) lattice of the $\mathrm{Ag}(100)$ substrate as evidenced by low-energy electron diffraction (LEED) results discussed elsewhere. ${ }^{17}$

Figure 1 illustrates the angle resolved photoemission spectra taken at normal emission for disordered 1 [Fig. 1(a)] and 2 [Fig. 1(b)] ML $\mathrm{Hg}$ overlayers on $\mathrm{Ag}$ ( 100). The $\mathrm{Hg}$ was deposited with the substrate temperature at $30 \mathrm{~K}$; the result was the formation of disordered solid $\mathrm{Hg}$ overlayers. These overlayers were subsequently and repeatedly annealed at 160 to $200 \mathrm{~K}$ and quenched to $30 \mathrm{~K}$ with no apparent change in the photoemission spectra taken following the requenching to $30 \mathrm{~K}$. The spectra for the "melted" $\mathrm{Hg}$ overlayers annealed at 160 to $200 \mathrm{~K}$ are also shown in Fig. 1. The LEED patterns of these $\mathrm{Hg}$ overlayers were indicative of a surface with considerable disorder and confirmed the lack of long-range crystallographic order for both the solid (adsorbed at $30 \mathrm{~K}$ ) and liquid overlayers.

The liquid overlayers exhibit photoemission spectra qualitatively different from well-ordered $\mathrm{Hg}$ overlayers. The electron density of states, in the region of $6 \mathrm{to} 7 \mathrm{eV}$ binding energy, between the $5 d \mathrm{Hg}$ shallow core levels and the $\mathrm{Ag}(100) d$ band (at $\sim 5-\mathrm{eV}$ binding energy) increased upon melting with the $\mathrm{Ag} d$-band intensity decreasing. This change is reversible up to ten annealing cycles (the largest number performed), for both 1 and $2 \mathrm{ML}$ of $\mathrm{Hg}$ adsorbed on Ag (100).

The $\mathrm{Hg}$ overlayers continue to "wet" the surface upon melting. If clumping or island formation (or indeed even alloying) accompanied the melting of the $\mathrm{Hg}$ overlayer, then the substrate Ag photoemission signal should increase, not decrease as observed. In addition, the changes in the photoemission features with temperature are reversible, arguing against substantial overlayer structural changes.

When the $\mathrm{Hg}$ overlayer is melted, not simply disordered, we expect the $\mathrm{Hg}$ atoms to occupy a variety of sites (in contrast to the thermodynamically most stable sites). This causes the broadening of the photoemission features observed, from the $\mathrm{Hg}$ overlayer adsorbed at 30 or $90 \mathrm{~K}$, as indicated by the results in Fig. 1. These results demonstrate that the electronic band structure of the disordered and melted overlayers differ and indicate the presence of local crystallographic order in the disordered overlayers.

Several studies of amorphous material ${ }^{20}$ have established that the nearest-neighbor and next-nearest-neighbor spacings and orientations remain predominantly the same for amorphous materials and their crystalline counterparts. Similarly, because $\mathrm{Hg}$ atoms adsorbed at $30 \mathrm{~K}$ will most likely occupy the most stable sites, which are the same sites occupied by $\mathrm{Hg}$ atoms in the ordered overlayer formed with adsorption at $90 \mathrm{~K}$, the principle difference in adsorption of $\mathrm{Hg}$ at 90 and $30 \mathrm{~K}$ is the long-range crystallographic order of the $\mathrm{Hg}$ thin film. Neither the disordered solid $\mathrm{Hg}$ overlayer nor the melted $\mathrm{Hg}$ overlayer exhibit an electronic density of states containing the same features as those observed in the spectra of the ordered $p(1 \times 1)$ of 1 and $2 \mathrm{ML}$ of $\mathrm{Hg}$ formed at $90 \mathrm{~K}^{14,17}$ (as seen in Fig. 1). The new electronic states (highlighted with an arrow in the inset to Fig. 1) are a consequence of the strained $\mathrm{Hg}$ ordered overlayer structure. ${ }^{2.13,21}$ There is no observation of similar electronic states in the disordered $\mathrm{Hg}$ overlayers adsorbed at $30 \mathrm{~K}$. This implies that long-range order is necessary for such states. Because the disorder of the overlayers formed at $30 \mathrm{~K}$ is largely going to be a result of defects, we conclude that short-range crystallographic order is not sufficient for the formation of such new $\mathrm{Hg}$ electronic states, since the local geometry about each atom is little different between adsorption at 30 and 90 K.

Judging from the experimental observations of the total density of states, one would expect the electronic density of states, broadly speaking, to remain similar. ${ }^{22-25}$ The present results therefore suggest one of two unexpected possibilities: the nearest-neighbor crystallography differs significantly for ordered and amorphous films of $\mathrm{Hg}$, or local density of states models for band structure are not applicable. ${ }^{22}$ Indeed, the change in the electron density of states is far greater than expected from even a change in lattice constant ${ }^{2,21}$ or the crystallographic symmetry.

Since the photoemission cross section of the $\mathrm{Hg}$ core levels 
are generally regarded as atomic in origin, the $\mathrm{Hg} / \mathrm{Ag}(100)$ system is particularly suited to investigate solid-state effects on the cross section in detail. Figure 2 illustrates a direct comparison of a disordered $5 \mathrm{ML} \mathrm{Hg}$ overlayer (open circles), a well-ordered 5-ML Hg overlayer (crosses with solid line), ${ }^{15}$ and the gas phase results ${ }^{26,27}$ (broken lines). These various cross sections in Fig. 2 have been normalized to be equal at $52 \mathrm{eV}$. This figure demonstrates that the disordered $\mathrm{Hg}$ overlayer exhibits a partial cross section quite similar to that observed for gaseous $\mathrm{Hg}^{26-35}$ and distinct from the partial cross sections attributable to the well-ordered $\mathrm{Hg}$ overlayer. ${ }^{15}$ The cross section of the disordered overlayers does not appear to be sensitive to the thickness of the $\mathrm{Hg}$ overlayer. The partial cross sections, therefore, depend on the long-range crystallographic order. We also note that for the disordered $\mathrm{Hg}$ overlayer, there is no indication of the narrow [full width at half-maximum (FWHM) approximately 1 $\mathrm{eV}]$ resonance so prominent in the well-ordered $\mathrm{Hg}$ overlayer. ${ }^{15}$

While recent theoretical calculations for $\mathrm{Hg}$ and $\mathrm{Rn}$ suggest that heavy metal partial cross sections of $\mathrm{Hg}$ are subject to the $E f$-wave potential, ${ }^{36}$ the cross section has never been demonstrated to depend upon long-range crystallographic order. Since both the sharp ( $1-2 \mathrm{eV}$ full width at half-maximum) cross-section resonance feature at $59 \mathrm{eV}$ and the broad featured (35-45 eV full width at half-maximum) about $65 \mathrm{eV}$ are altered by the crystallographic order, photoemission diffraction effects cannot be used to explain all of our observed cross-section changes. Photoemission diffraction can alter the partial photoemission cross sections ${ }^{37.38}$ only at specific photon energies, and result in changes in the cross section only over small $(-2 \mathrm{eV})$ photon energy regions. The necessary conditions ${ }^{37,38}$ for such photoelectron diffraction are not satisfied. A change not in the local order, but in the long-range crystallographic order must, therefore, somehow have a profound effect upon the electronic structure of the $\mathrm{Hg}$ overlayer.

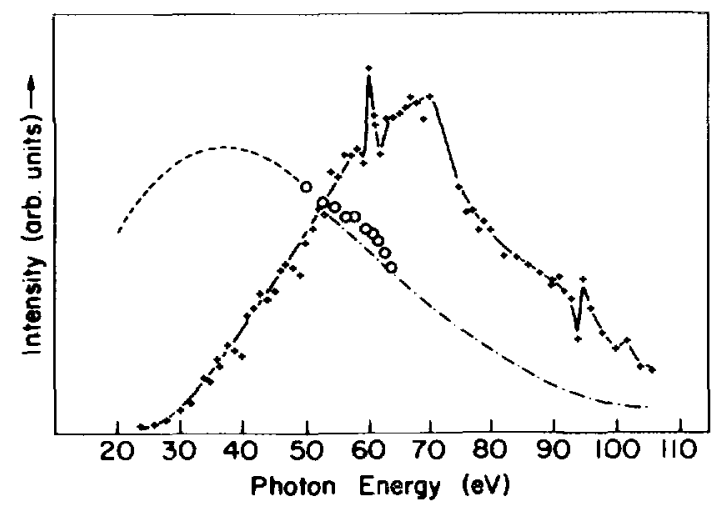

FiG. 2. The relative photoemission cross section of the $\mathbf{H g} 5 d_{5,2}$-like orbital for a 5 monolayer (13-14 L) thin film of $\mathrm{Hg}$ on $\mathrm{Ag}(100)$ at $30(\mathrm{O})$ and $87 \mathrm{~K}$ $(+)$. The relative cross sections were compiled from EDC's taken with the collection angle normal to the surface and the vector potential of the light parallel with the surface. The gaseous mercury cross section is shown as $(\ldots)$ from Ref. 26 and (...) from Ref. 27.
To confirm that defects in the long-range crystallographic order affect the $\mathrm{Hg} 5 d$ partial cross sections, we deposited 20 $\mathrm{L}$ ( $7 \mathrm{ML}$ ) of $\mathrm{Hg}$ on $\mathrm{Ag}(100)$ held at $70 \mathrm{~K}$ and produced a disordered $\mathrm{Hg}$ overlayer with no LEED pattern, and with only a weak feature in the photoemission attributable to the third $5 d \mathrm{Hg}$ feature. We measured the partial cross sections of the $5 d$-like states for the photon energy range $50 \mathrm{eV}$ $\leqslant h v \leqslant 60 \mathrm{eV}$. We annealed the sample gently ( to $\sim 100 \mathrm{~K}$ ) and cooled the sample back to $70 \mathrm{~K}$ after each annealing treatment.

As the overlayer is annealed, the long-range order increases, as demonstrated by LEED, and the partial cross section of the three $5 d$-like $\mathrm{Hg}$ states changes. Since the coverage does not change, the cross-section changes can only be due to changes in the structure. The change in the cross section is precisely the expected: as the defect density is reduced by annealing, and the long-range order increases, the cross section changes from that characteristic of gas phase $\mathrm{Hg}$ (decreasing with photon energy) to that of the well-ordered $\mathrm{Hg}$ overlayer (increasing with photon energy). The cross section of all three $\mathrm{Hg} 5 d$ states depends upon longrange crystallographic order of the $\mathrm{Hg}$ overlayer.

The 2- and 5-ML overlayers exhibit both well-ordered and disordered overlayers. The new electronic state was present in the photoemission spectra of the well-ordered overlayers and absent for the disordered overlayers. Figure 3 illustrates the branching ratio for well-ordered 2-ML (crosses) and disordered 2-ML overlayers (open circles) obtained by depositing $\mathrm{Hg}$ onto a $\mathrm{Ag}(100)$ sample held at 90 and $30 \mathrm{~K}$, respectively, in the photon energy range from 50 to $70 \mathrm{eV}$. Note that the disordered 2-ML overlayers closely resembles the gas phase $\mathrm{Hg}$ results. ${ }^{28.29 .39}$ The well-ordered $\mathrm{Hg}$ overlayers exhibit a significantly higher branching ratio by comparison, some $10 \%$ to $40 \%$ higher than the gas phase results.

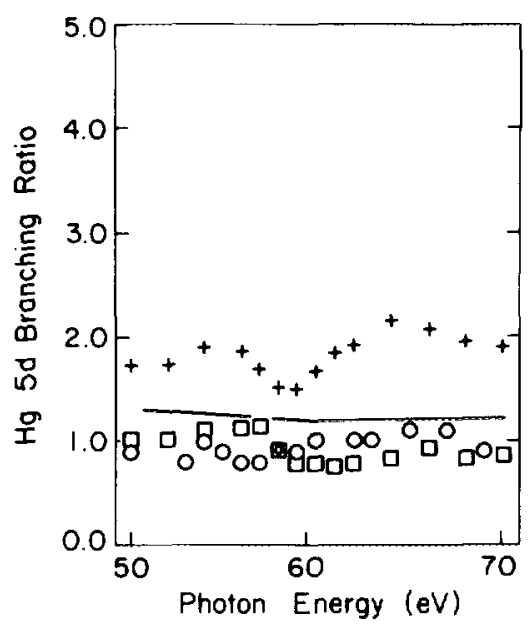

FIG. 3. The branching ratio for the $\mathrm{Hg} 5 d$ states as a function of photon energy for $2 \mathrm{ML}$ of $\mathrm{Hg}$ adsorbed on $\mathrm{Ag}(100) .(+)$ represents the $5 d$ ratio for an ordered overlayer adsorbed at $90 \mathrm{~K}$ and with the third $5 d \mathrm{Hg}$ feature included in the ratio, while the $(\square)$ symbols indicate the ratio for this overlayer without incorporation of the third feature intensity. $(O)$ indicates the $5 d_{5,2}$ to $5 d_{3 / 2}$ ratio for a disordered $2 \mathrm{ML}$ overlayer of $\mathrm{Hg}$ on $\mathrm{Ag}(100)$ at 30 $\mathrm{K}$. The solid line is the observed branching ratio for gaseous $\mathrm{Hg}$ (Refs. 28, 29 , and 39 ). 
The branching ratio obtained for the well-ordered and disordered 5-ML $\mathrm{Hg}$ overlayers are illustrated in Fig. 4. We observed a dramatic difference between the well-ordered overlayer (crosses) and a disordered $\mathrm{Hg}$ overlayer of equal thickness (open circles) as shown in Fig. 4. The well-ordered $\mathrm{Hg}$ overlayer exhibits two maxima in the branching ratio, a sharp ( $F W H M=4 \mathrm{eV}$ ) maximum of 7.2 at a photon energy of $28 \mathrm{eV}$ and a broad maximum of 2.6 at $\sim 68-\mathrm{eV}$ photon energy. The gas phase results $(\triangle)$ of Refs. 28,29 , and 39 exhibit corresponding branching ratio of $\sim 1.7$ and $\sim 1.4$ at photon energies of 28 and $68 \mathrm{eV}$, respectively. The disordered $\mathrm{Hg}$ overlayer exhibits a branching ratio similar to that of gas phase $\mathrm{Hg}$, differing by not more than $15 \%$ from the statistical value except at photon energies less than 25 $\mathrm{eV}$. This result, of a comparatively high branching ratio at photon energies below $25 \mathrm{eV}$, has been previously reported for $\mathrm{HgS}^{4(1)}$

Taking long-range crystallographic order into account in estimating the branching ratio is clearly necessary, as Figs. 3 and 4 demonstrate. The branching ratio of the disordered $\mathrm{Hg}$ overlayers resembles the gas phase branching ratio, while the deviation in the branching ratio is clearly apparent when the thin $\mathrm{Hg}$ overlayer is ordered. The difference between the two overlayers must lie in the presence or absence of longrange crystallographic order.

Photoemission diffraction can alter the partial photoemission cross sections $\mathrm{s}^{37,38}$ and as a consequence can alter the branching ratio, but such effects, as mentioned previously, can be excluded. It is also worth noting that the large deviations of the branching ratio from the statistical value of 1.5 observed at 28 and $58 \mathrm{eV}$ not only cover too broad a photon energy range to be explained by photoelectron diffraction, but do not correspond to features in the partial cross sections characteristic of photoelectron diffraction (Fig. 2).

The presence of the new electronic state demonstrably and significantly affects the branching ratio. When the influence of the new electronic state is removed either by our data analysis software or by growing disordered overlayers, the branching ratio more closely approaches that observed for gaseous $\mathrm{Hg}$. An understanding of the origin of the new third $5 d \mathrm{Hg}$ state appears essential to developing any understand-

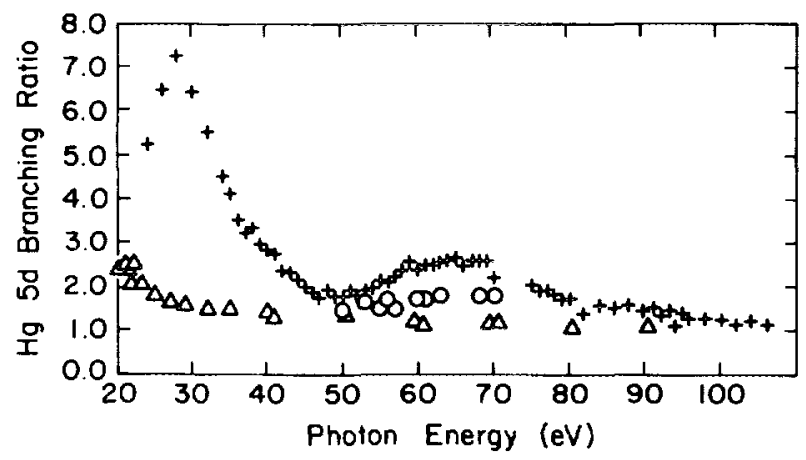

Fig. 4. The branching ratios of the $\mathrm{Hg} 5 d$ states for a 5-ML-thick overlayer of $\mathrm{Hg}$ on $\mathrm{Ag}(100)$ as a function of photon energy. ( + ) shows the ratio for an ordered overlayer and the third-feature intensity included in the ratio. $(\triangle)$ shows the ratio for gaseous monoatomic $\mathrm{Hg}$ (Refs. 28, 29, and 39). $(O)$ shows the results for a disordered overlayer. ing of the observed large deviations of the branching ratio for the ordered $\mathrm{Hg}$ overlayers from those branching ratios observed with gaseous $\mathrm{Hg}$ and disordered overlayers. There is little doubt that this feature is a consequence of the electronic structure of the $\mathrm{Hg}$ overlayer. ${ }^{17}$ Furthermore, this third feature is not a result of the electronic structure of the $\mathrm{Ag}(100)$ substrate since the feature does not attenuate in intensity with increasing overlayer thickness, ${ }^{14,17}$ is observed for $\mathrm{Hg}$ overlayers on other metal substrates, ${ }^{1,16}$ and cannot be reconciled with the electronic structure of the $\mathrm{Ag}(100)$ substrate. ${ }^{17}$ The decrease of the partial $5 d_{3 / 2}$ and $5 d_{5 / 2}$ cross sections (neglecting the new state) in the transition from disordered to ordered $\mathrm{Hg}$ overlayers suggests that the new $5 d$ state is made of approximately one electron from the $5 d_{3 / 2}$ state and one electron from the $5 d_{5 / 2}$ state. The apparent contributions of the $5 d_{5 / 2}$ and $5 d_{3 / 2}$ states to the new $\mathrm{Hg}$ $5 d$ state oscillator strength are consistent with the general deviation of the branching ratios, observed for the ordered 5 and 2-ML $\mathrm{Hg}$ overlayers, of 2.3 as compared to the more statistical value of 1.5 approximated by the disordered overlayers and gaseous $\mathrm{Hg}$. Such a shift in oscillator strength is not consistent with "standard" bonding-antibonding molecular-orbital-type models of hybridization. Such a shift in electron distribution is suggestive of a change in the spinorbit interaction.

Changes in the spin-orbit interaction can result in substantial changes in the $5 d$ branching ratio. ${ }^{41}$ Unfortunately, changes in the $5 d_{5 / 2}$ to $5 d_{3 / 2}$ spin-orbit binding-energy splitting expected with a change in the spin-orbit interaction ${ }^{41}$ are not observed. ${ }^{1.2 .16}$ Furthermore, there is no reason to expect a large change in the electron occupancy of $5 d$ states $7-10 \mathrm{eV}$ below the Fermi energy, particularly when this change must be greater than is observed for a compound such as $\mathrm{HgS},{ }^{40}$ nor is it clear why the spin-orbit interactions (a largely intra-atomic interaction) should be affected by long-range crystallographic order (indicating an extraatomic many-electron interaction).

The overall $\mathrm{Hg} 5 d_{5 / 2}$ and $5 d_{3 / 2}$ partial cross sections for well ordered $\mathrm{Hg}$ overlayers have the cross-section maximum shifted to higher photon energies and the cross-section maximum is broader than is the case for gaseous $\mathrm{Hg}^{22.23}$ Existing calculations, ${ }^{42}$ although only considering the nearest-neighbor interactions, have indicated that such a shift in the broad maximum in the photoemission cross section is consistent with a change in the exchange-correlation potential for the interaction between a given atomic orbital and the surrounding atoms, from a localized one-electron, seif-consistentfield potential to a potential accommodating essentially freeelectron states. In the example reported here, for the well-ordered $\mathrm{Hg}$ overlayers, the $\mathrm{Hg} 5 d$ levels appear to interact in a manner consistent with a many-electron exchange and correlation potential. Noting that the cross section of the $5 d \mathrm{Hg}$ levels depends upon long-range crystallographic order of the overlayer, the implication is that the $\mathrm{Hg} 5 d$ interaction extends beyond the second nearest neighbor.

\section{CONCLUSION}

In summary, we have observed three types of qualitative changes in the photoemission cross section of well-ordered 
$\mathrm{Hg}$ overlayers on $\mathrm{Ag}(100)$ as compared to gas phase $\mathrm{Hg}$ and have established the essential role of long-range crystallographic order upon the electronic stucture, a heretofore unreported relationship. Long-range order affecting the electronic structure of semiconductors has been postulated ${ }^{43}$ but without the clear evidence presented here. We have also demonstrated the necessity for long-range crystallographic order for observing the new $\mathrm{Hg}$ overlayer electronic states. The change in the density of states is a dramatic change, and cannot be explained by a simple broadening of the local density of states. We have thus established that the presence or absence of long-range crystallographic order influences the photoemission cross section of $\mathrm{Hg}$ electronic states 8 and 10 $\mathrm{eV}$ below the Fermi energy, commonly viewed as shallow core levels.

\section{ACKNOWLEDGMENTS}

Financial support for this research was provided by the National Science Foundation through Grants No. DMR83-04368 and No. DMR-86-57109 and by AFOSR Task No. 2305J9 monitored under RADC postdoctoral program Contract No. F30602-88-D-0027. The work was performed at the University of Wisconsin Synchrotron Radiation Cen$\operatorname{ter}$ (SRC) which is supported by the National Science Foundation through Grant No. DMR-86-01349.

\footnotetext{
"NSF Presidential Young Investigator, affiliated with the University of Texas for portions of this work.

'P. A. Dowben, M. Onellion, and Y. J. Kime, Scan. Microsc. 2, 177 (1988). 'Shikha Varma, Y. J. Kime, P. A. Dowben, M. Onellion, and J. L. Erskine, Phys. Rev. (submitted)

${ }^{3}$ M. Onellion, P. A. Dowben, and J. L. Erskine, Phys. Lett. A 130, 171 (1988).

${ }^{4}$ D. W. Lynch and J. H. Weaver, "Photoemission of Ce and its Compounds," in Handbook on the Physics and Chemistry of Rare Earths, edited by K. A. Gschneidner, Jr.. L. Eyring, and S. Hufner (North-Holland, Amsterdam, 1987), Vol. 10.

'Y. Baer and W.-D. Schneider, "High Energy Spectroscopy of Lanthanide Materials: An Overview," in Ref. 4.

"D. M. Wieliczka, C. G. Olson, and D. W. Lynch, Phys. Rev. Lett. 52, 2180 (1984).

'D. M. Wieliczka, J. H. Weaver, D. W. Lynch, and C. G. Olson, Phys. Rev. B 26, 7056 (1982).

${ }^{*}$ D. M. Wieliczka, C. G. Olson, and D. W. Lynch, Phys. Rev. B 29, 3028
}

(1984).

${ }^{9}$ W.-D. Schneider, C. Laubschat, and B. Reihl, Phys. Rev. B 27, 6538 (1983).

${ }^{10}$ F. Gerken, A. S. Foldstrom, J. Barth, L. I. Johansson, and C. Kunz, Phys. Scr. 32, 43 (1985).

${ }^{11}$ H. F. J. Jansen, A. J. Freeman, M. Weinert, and E. Wimmer, Phys. Rev. B 28, 593 (1983).

${ }^{12}$ A. R. Miedema and J. W. F. Dorleijn, Philos. Mag. B 43, 251 (1981).

${ }^{13}$ P. A. Dowben, Shikha Varma, Y. J. Kime, D. R. Mueller, and M. Onellion, Z. Phys. B 73, 247 (1988).

${ }^{14}$ M. Onellion, J. L. Erskine, Y. J. Kime, Shikha Varma, and P. A. Dowben, Phys. Rev. B 33, 8833 (1986).

${ }^{15}$ Shikha Varma, Y. J. Kime, P. A. Dowben, M. Onellion, and J. L. Erskine, Phys. Lett. A 116, 66 (1986).

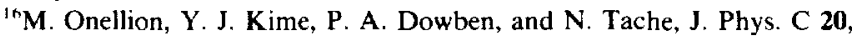
L633 (1987).

${ }^{17}$ P. A. Dowben, Y. J. Kime, Shikha Varma, M. Onellion, and J. L. Erskine, Phys. Rev. B 36, 2519 (1987).

${ }^{18}$ S. Svensson, N. Martensson, E. Basilier, P. A. Malmqvist, U. Gelius, and K. Siegbahn, J. Electron. Spectrosc. Relat. Phenom. 9, 51 (1976).

${ }^{19}$ R. W. Joyner, M. W. Roberts, and K. Yates, Surf. Sci. 87, 501 (1979).

${ }^{20}$ N. F. Mott and E. A. Davis, Electronic Processes in Non-Crystalline Materials, 2nd Ed. (Clarendon, Oxford, 1979).

${ }^{2}$ 'Shikha Varma, Y. J. Kime, P. A. Dowben, and M. Onellion, Proc. Mat. Res. Soc. (1989) (in press).

${ }^{22}$ W. A. Harrison, Electronic Structure and Properties of Solids (Freeman, San Francisco, 1980).

${ }^{23}$ J. A. Catterall and J. Trotter, Philos. Mag. 8, 897 (1963).

${ }^{24}$ D. E. Eastman, Phys. Rev. Lett. 26, 1108 (1971).

"s. W. Spicer, in Band Structure of Metals and Alloys, edited by D. J. Fabian and L. M. Watson (Academic, New York, 1973).

${ }^{26 J}$ J. L. Dehmer and J. Berkowitz, Phys. Rev. A 10, 484 (1974).

${ }^{27}$ J. S. Shyu and S. T. Manson, Phys. Rev. A 11, 166 (1975).

${ }^{2 K}$ S. P. Shannon and K. Codling, J. Phys. B 11, 1193 (1978).

${ }^{20}$ P. H. Kobrin, P. A. Heimann, H. G. Kerkhoff, D. W. Lindle, C. M. Truesdale et al., Phys. Rev. A 27, 3031 (1983).

${ }^{31}$ F. Keller and F. Combet Farnoux, J. Phys. B 12, 2821 (1979).

${ }^{31}$ T. E. H. Walker and J. T. Waber, J. Phys. B 7, 674 (1974).

${ }^{32}$ F. Keller and F. Combet Farnoux, J. Phys. B 15, 2657 (1982).

${ }^{3.3}$ W. R. Johnson, V. Radojevic, P. Deshmukh, and K. T. Cheng, Phys. Rev. A 25, 337 (1982).

${ }^{34}$ B. R. Tambe, W. Ong, and S. T. Manson, Phys. Rev. A 23, 799 (1981)

${ }^{35}$ W. R. Johnson and V. Radojevic, Phys. Lett. A 92, 75 (1982).

${ }^{36}$ B. R. Tambe and S. T. Manson, Phys. Rev. A 30, 256 (1984).

"P. A. Dowben, D. Heskett, E. W. Plummer, Y. Sakisaka, T. N. Rhodin, and C. Umrigar, Phys. Rev. Lett. 53, 1493 (1984).

${ }^{36}$ T. C. Hsieh, P. John, T. Miller, and T. C. Chiang, Phys. Rev. B 35, 3728 (1987).

${ }^{34}$ S. Süzer, S. T. Lee, and D. A. Shirley, Phys. Rev. A 13, 1842 (1976).

${ }^{40}$ G. Margaritondo, R. Rosei, J. H. Weaver, and W. M. Becker, Solid State Commun. 34, 401 (1980)

${ }^{4 !}$ G. van der Laan and B. T. Thole, Phys. Rev. Lett. 60, 1977 (1988)

${ }^{42}$ S. T. Manson, in Photoemission in Solids I, edited by M. Cardona and L. Ley (Springer, Berlin, 1978), pp. 135-164.

${ }^{43}$ R. S. List, J. C. Wolcik, I. Lindau, and W. E. Spicer, J. Vac. Sci. Technol. B 5, 1279 (1987). 